\title{
Role of Three-Dimensional Ultrasound in Gynecology
}

\author{
Turkgeldi Engin $\cdot$ Urman Bulent $\cdot$ Ata Baris
}

Received: 16 September 2014/ Accepted: 7 October 2014/Published online: 8 November 2014

(C) Federation of Obstetric \& Gynecological Societies of India 2014

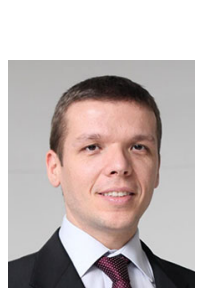

\section{About the Author}

Dr. Engin Türkgeldi graduated from Cerrahpaşa Faculty of Medicine of Istanbul University in 2004. He completed his Obstetrics and Gynecology residency in 2010. He worked at the American Hospital in Istanbul, and attended a certification program on "Infertility and Assisted Reproductive Techniques" at the same department. He is currently working in Koç University Hospital in Istanbul.

\begin{abstract}
Three-dimensional ultrasound (3D USG) is a fast-evolving imaging technique that holds a great potential for use in gynecology. Its sensitivity and specificity is reported to be close to $100 \%$ for diagnosing congenital uterine anomalies, comparable with those of magnetic resonance imaging (MRI) and laparoscopy. With 3D USG, a coronal view of the uterus can be obtained, clearly outlining the external contour of the uterus and providing accurate information about the shape of the cavity. Although 3D USG may not perform well in thin endometria, combining it with saline infusion sonography (SIS) overcomes this problem. Research shows that 3D USG is more sensitive and specific than two-dimensional ultrasound (2D USG) in defining and mapping uterine lesions,
\end{abstract}

Turkgeldi E. · Urman B. · Ata B. ( $\square)$

Department of Obstetrics and Gynecology, Amerikan Hospital, Koc University School of Medicine, Rumelifeneri Yolu Sarıer, 34450 Istanbul, Turkey

e-mail: barisata@ku.edu.tr such as fibroids, adenomyosis, and intrauterine synechia. In cases of suspected malignancy, 3D USG is mainly used in the initial evaluation of patients. Measuring various indices and mapping vascular architecture with 3D power Doppler have been proposed for evaluating adnexal masses. Although some studies raised hope, no consensus is reached about its use, success, and limitations. In urogynecology, translabial 3D USG is proved to be a valuable tool, as it provides instant access to the axial plane, which clearly depicts the relationship of the vagina, urethra, rectum, and the muscular pelvic floor. Studies report no significant differences between translabial 3D USG and MRI measurements for evaluation of the pelvic floor. In conclusion, adding 3D USG to routine gynecological workup can be beneficial for clinicians, as it provides fast and accurate results in a relatively cost-effective setting.

Keywords Three-dimensional ultrasound .

Congenital uterine anomalies · Gynecology · Oncology · Urogynecology 


\section{Introduction}

Three-dimensional ultrasound (3D USG) technology, that has been developed three decades ago, was initially used in obstetrics; however, its success raised interest about its potential in gynecology. Studies so far demonstrated that 3D USG can be a useful and, in some areas, an indispensible tool in evaluation of the gynecology patient.

As in two-dimensional ultrasound (2D USG), transvaginal approach is preferred in gynecologic examination with 3D USG. The transducer is held still close to the area of interest, and a quick sweep at the desired angle is performed. Acquired data are used to obtain a volume. The electronically stored data are processed to display three orthogonal planes. These planes can be rotated or moved as desired, and can be united to form a single 3D image. Manipulation of the acquired volume data is possible to examine a specific structure, such as surface rendering for evaluating congenital uterine anomalies. Another option is to view the volume in slices, as in computerized tomography. This is called multi-slice imaging. The number of slices and the space between them are user-defined.

The data volume, which is acquired in seconds while sweeping, can be stored and reviewed later. This shortens the examination time and makes it possible to manipulate and review the data even in the absence of the patient. It can also be used for consulting colleagues, auditing, and educational purposes.

An outstanding property of 3D USG is its capability to obtain images in previously unavailable planes using $2 \mathrm{D}$ USG. Coronal plane can provide detailed information about uterus and adnexa, and it is especially helpful in diagnosing congenital uterine anomalies (Fig. 1). Likewise, pelvic floor can be assessed efficiently in axial plane using 3D USG. Moreover, the user is not limited by these conventional planes. It is possible to construct any plane from the acquired volume data.

This review will focus on the clinical applications of 3D USG in gynecology. Details about specific scanning techniques, device settings, and data management methods are beyond the scope of this review.

\section{Congenital Anomalies}

Congenital uterine anomalies arise due to unsuccessful development and/or fusion of Müllerian ducts or failure in resorption of uterovaginal septa. Prevalence of congenital uterine anomalies is reported to be $5.5 \%$ in the general population, $8 \%$ in the infertile population, and $13.3 \%$ in women with recurrent miscarriages [1]. The relationship of these anomalies with infertility and adverse pregnancy outcomes is well documented. Septate uterus, the most

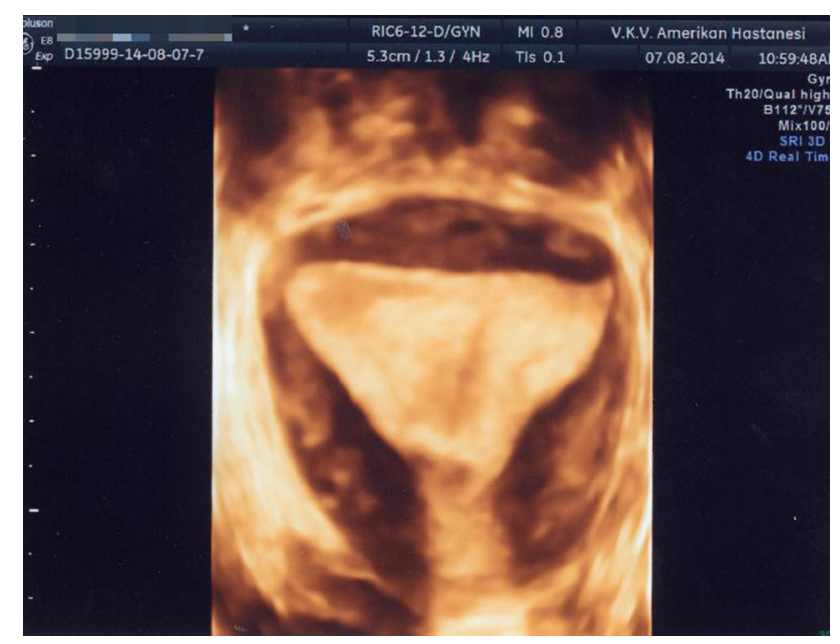

Fig. 1 Three-dimensional image of a normal uterus. The coronal view shows both the uterine cavity and external contour of the uterus clearly

common uterine anomaly, is associated with infertility and spontaneous abortion [2]. Unification defects, namely unicornuate, bicornuate, and didelphic uterus, increase the risk for abortion, preterm labor, and fetal malpresentation. Once considered a minor defect, arcuate uterus is found to increase the risk for second trimester pregnancy losses and preterm labor [3].

2D USG and hysterosalpingography (HSG) have been the two traditional methods used in the investigation of congenital uterine anomalies. While a good quality HSG can provide accurate information about the uterine cavity, it cannot evaluate the external contour of the uterus. Moreover, it is an invasive procedure that requires the presence of a gynecologist or a radiologist. Sometimes an anesthesiologist is needed, as it can be painful for the patient. In their review, Saravelos et al. reported HSG's sensitivity, specificity, and accuracy in diagnosing uterine anomalies to be 78,90 , and $86 \%$, respectively (weighted mean of 9 studies) [2]. Recently, 2D USG's power to detect uterine anomalies has been enhanced by saline infusion sonography (SIS), in which saline is infused to distend the uterine cavity. This method gives satisfactory information on the shape of the cavity. However, its inability to assess the external contours is a drawback. 2D SIS increased 2D USG's sensitivity from 56 to $93 \%$, accuracy from 84 to $97 \%$, and maintained its specificity at $99 \%$ (weighted mean of 5 and 7 studies, respectively) [2].

Magnetic resonance imaging (MRI) is a non-invasive imaging modality for diagnosing uterine anomalies. It can examine both the uterine cavity and the external contour of the uterus. Yet it is costly, time consuming, and not widely available. The presence of nearby bowel movements and a retroflexed uterus hinders MRI evaluation [4]. It cannot always be interpreted by a radiologist experienced in uterine 


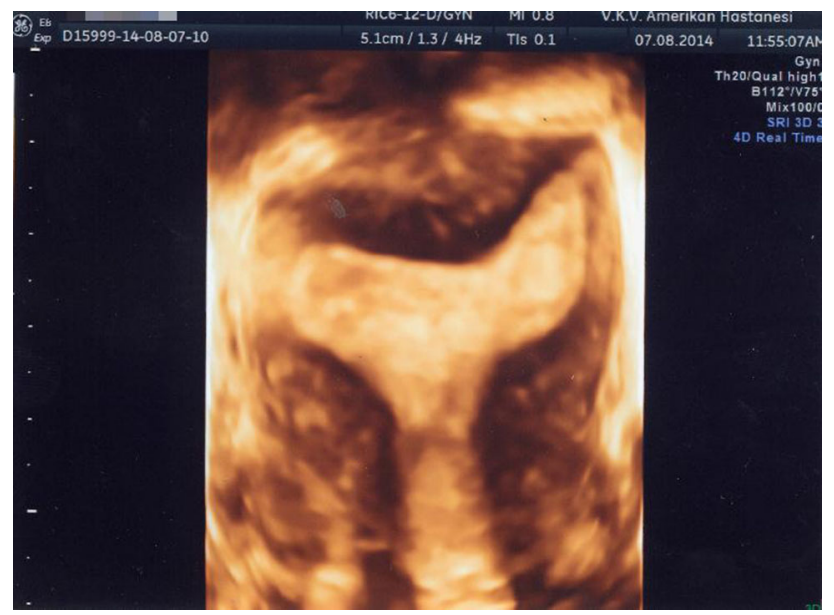

Fig. 2 Three-dimensional image of an arcuate uterus. Please note that while the external contour of the uterus is normal, there is mild fundal indentation in the cavity

anomalies, which can reduce its accuracy [5]. The gold standard in the diagnosis of congenital uterine anomalies is laparoscopy performed concurrently with hysteroscopy. Despite being highly specific and accurate, laparoscopy is an invasive procedure with potential morbidity, making it hard to justify its use solely for diagnostic purposes.

In recent years, 3D USG has proved to be a very powerful tool for diagnosing uterine anomalies. The most important advantage of 3D USG for diagnosing uterine anomalies is its ability to obtain a coronal view of the uterus, which is not possible in 2D USG due to the bony pelvis. This view clearly outlines the external contour of the uterus, as well as providing accurate information about the shape of the cavity (Figs. 2, 3). Ideally, the endometrium should be at least $5 \mathrm{~mm}$ thick during the examination, as the benefit of the $3 \mathrm{D}$ coronal view is significantly reduced in thinner endometria [6].

Faivre et al. [5] and Ghi et al. [7] have reported $100 \%$ specificity and sensitivity for 3D USG, and a concordance of 100 and $96 \%$, respectively, when compared with laparoscopy and concurrent hysteroscopy. Faivre reported that 3D USG had higher concordance with laparoscopy than MRI. Another study examined 65 patients with suspected uterine anomalies, using both 3D USG and MRI. It showed a high degree of concordance between 3D USG and MRI (kappa index 0.880), and stated that the few differences that were observed were in the evaluation of lower uterine parts [8]. Ata et al. demonstrated that 3D USG not only had $100 \%$ categorical agreement with MRI for diagnosing uterine septa, but its measurements of septum length, septum width, and septum-serosa distance were also in concordance with those of MRI [9].

Recently, the use of 3D USG in combination with SIS has been investigated. In their study of 117 patients,

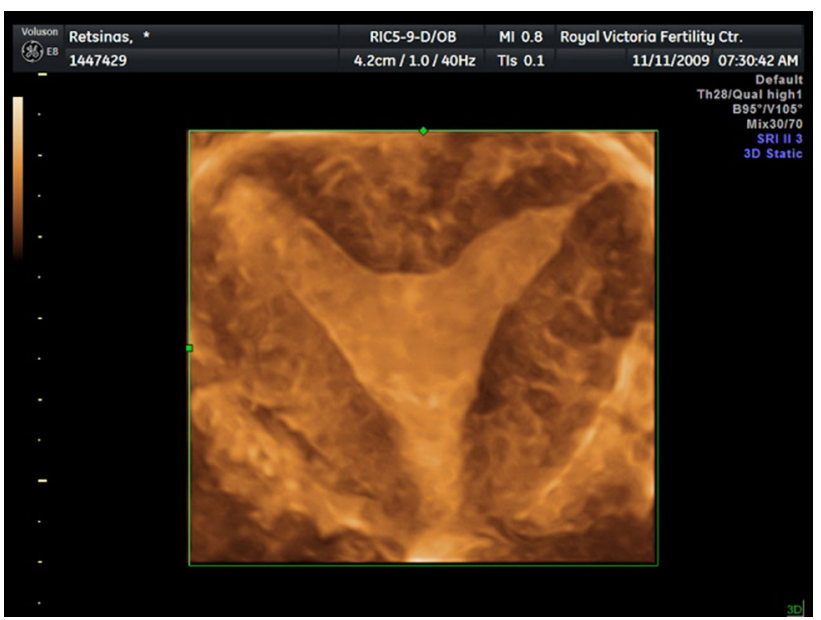

Fig. 3 Three-dimensional image of a septate uterus. The coronal view clearly depicts the normal external contour of the uterus and the deeply indented fundal part of the cavity

Ludwin et al. compared 3D SIS, 3D USG, and 2D USG. 3D SIS had $100 \%$ accuracy, sensitivity and specificity, and it was significantly better than the others in diagnosing uterine anomalies. It was the only modality that was $100 \%$ consistent with hysteroscopic and laparoscopic findings [10]. Another benefit of 3D SIS is that it does not require the endometrium to be thicker than $5 \mathrm{~mm}$. In another study of 141 patients, 3D SIS was also found to have $97.2 \%$ sensitivity for the overall evaluation of the shape of the uterine cavity, fundal notch, and intrauterine adhesions. According to these results, the need for second-look hysteroscopy is almost eliminated [11].

3D USG can be used not only for reaching a diagnosis but also for optimizing the treatment. Ludwin et al. used real-time 3D transrectal USG during hysteroscopic metroplasty for uterine septum. With this method, the surgeon knows exactly how much septum is left in real time. This helps in preventing overzealous incisions and reduces the risk of incomplete septum resection [12].

In summary, 3D USG is a highly sensitive and specific tool for accurately diagnosing congenital uterine anomalies. It is non-invasive, fast, reproducible, readily available, and relatively cost-effective. Over time, it is strongly possible that 3D USG may become the gold standard in diagnosing uterine anomalies, sidelining MRI. Hysteroscopy and laparoscopy may then be used for therapeutic purposes only.

\section{Benign Gynecology}

Leiomyomas, the most common benign tumor in women, are relatively easily diagnosed by gynecologists. However, with 2D USG it can be a challenge to differentiate a submucosal leiomyoma from an intramural one, or to 
determine its relationship with the endometrial cavity. This is especially important in patients with fertility problems, recurrent pregnancy failures, and abnormal uterine bleeding. Likewise, distinguishing the borders and exact location of multiple leiomyomas can be difficult with 2D USG. 3D USG can precisely map the location of leiomyomas and depict their intramural and submucosal components [4]. This information is very valuable when making clinical decisions.

3D power Doppler can also be useful in evaluating candidates for embolization. It gives detailed information about collateral vessels, the presence of which decreases the chance of success [13].

As in 2D USG, SIS improves the diagnostic power of 3D USG. SIS is especially useful when the endometrium is thin. In these patients, the contrast between the endometrium and myometrium is faint, making a diagnosis difficult [4]. Abou-Salem et al. compared the efficacy of 2D SIS, 3D SIS, and hysteroscopy in diagnosing the etiology of abnormal uterine bleeding. All three methods were performed on the same 70 women. They reported $92 \%$ sensitivity and $89 \%$ specificity for 3D SIS in diagnosing intrauterine lesions such as submucous myomas, endometrial polyps, and endometrial hyperplasia. These results were significantly better than 2D SIS and comparable to those of diagnostic hysteroscopy [14].

Intrauterine adhesions are a well-known cause of infertility. HSG and 2D SIS are the two common methods used for its diagnosis, while hystereoscopy is the golden standard. The role of 3D USG in the diagnosis has not been investigated widely. A study comparing 3D USG and HSG reported 3D USG to have a sensitivity of $100 \%$ and HSG $66.7 \%$ for correctly diagnosing and grading intrauterine adhesions. HSG failed in diagnosing lower uterine segment adhesions mostly, mistaking them for complete cavity obstructions [15]. Ahmadi acknowledged 3D SIS to be a useful, minimally invasive tool [16] (Figs. 4, 5).

The role of 3D USG in the diagnosis of adenomyosis has been investigated recently. The junctional zone, the disrupted border between the basal endometrial layer and the endometrium in patients with adenomyosis, is clearly visible in all planes in 3D USG. Exacoutos et al. reported that sensitivity of 3D USG (91\%) in diagnosing adenomyosis was significantly better than the that of 2D USG, but its specificity and accuracy was similar [17]. Luciano et al. examined 54 premenopausal women scheduled for hysterectomy using 3D USG, and compared the results with the histopathological findings. They stated that in patients with no history of endometrial ablation or medical treatment, presence of two or more 3D USG markers of adenomyosis yielded $90 \%$ accuracy, $92 \%$ sensitivity, and $83 \%$ specificity, which were comparable to MRI results. However, they noted that the accuracy of 3D USG

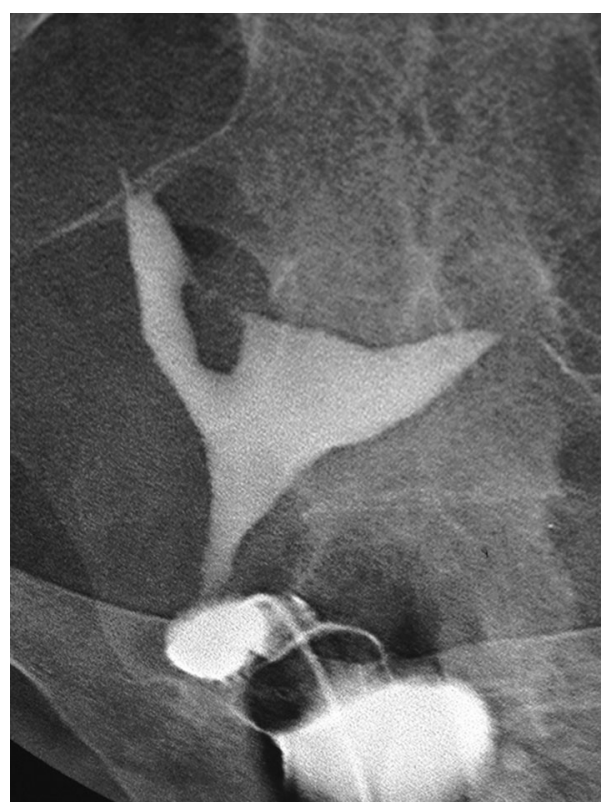

Fig. 4 Hysterosalpingography of intrauterine synechia

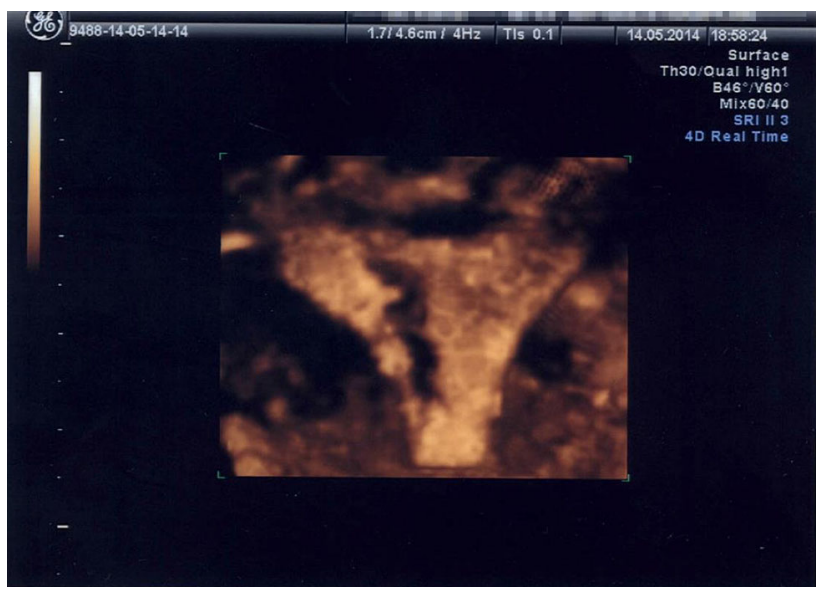

Fig. 5 Three-dimensional image of intrauterine synechia of the same patient. The synechia is seen as an irregular hypoechoic line starting from the right-fundal region, extending down to the isthmic level

decreased to $50 \%$ in patients who have undergone endometrial ablation or medical therapy [18]. This is probably due to loss of contrast in very thin endometria.

Intrauterine devices (IUDs) are used widely for contraception. Malpositioned IUDs or those embedded in the myometrium may cause abnormal bleeding and pelvic pain. 2D USG can usually show the shaft of the device; however, it may fail to show the side arms. The acoustic shadow of the shaft may make it difficult to observe the exact position of the IUD in the cavity. Moreover, levonorgestrel-releasing intrauterine devices do not have copper components and are harder to locate in the cavity. 
Coronal view of the uterus in 3D USG can clearly show the entire IUD, its location in the cavity, and its relationship with the myometrium [19].

When planning surgery for deep pelvic endometriosis (DIE), it is important to know the location and size of the endometriotic nodules, as well as their involvement with pelvic structures and organs. MRI and 2D USG are the most commonly used imaging modalities to determine these. Bazot et al. reported a sensitivity of $87 \%$ and an accuracy of $86 \%$ for DIE using transvaginal 2D USG [20]. Implementation of the "tenderness guided" 2D USG technique provided $95 \%$ specificity and $90 \%$ sensitivity [21]. Recently, Guerriero et al. investigated the role of 3D USG in DIE. They concluded that while the sensitivity and specificity of 2D USG and 3D USG were not statistically different for rectosigmoid involvement, 3D USG reduced false negatives by $47-75 \%$ and was significantly more specific in non-intestinal locations, namely the uterosacral ligaments, rectovaginal septum, and vaginal fornix [22].

3D USG technology not only improves on current 2D techniques, but also provides new applications, such as automated volume calculation. Sonography-based Automated Volume Calculation (SonoAVC, GE Medical Systems) is one of these applications, which identifies hypoechoic structures and calculates their volume from the acquired $3 \mathrm{D}$ dataset. It is mainly used for counting antral follicles and monitoring follicular growth in in vitro fertilization (IVF) cycles (Fig. 6). It is especially useful in patients with good ovarian reserve, in whom these two examinations take time and miscalculations are more likely. Measuring follicles using SonoAVC is found to reduce examination time significantly [23], which is invaluable in a busy IVF clinic setting. In their two studies, Ata et al. concluded that SonoAVC technology saves time, makes accurate measurements, and is highly reproducible. Its disadvantages are the need for post-processing and performance of occasional manual measurements [24, 25].

In conclusion, it may seem that there is not much to improve on 2D USG for diagnosing benign gynecological diseases, considering it is a widely used, effective and established imaging modality in gynecology. However, research shows that 3D USG is better in defining and mapping uterine lesions, and it is more sensitive and specific than 2D USG for a number of conditions. Implementing SIS to 3D USG increases its effectiveness even further, especially in patients with thin endometria. Adding 3D USG to their gynecological workup will aid clinicians in diagnosing and managing their patients with benign gynecological conditions.

\section{Oncology}

Ultrasound is the initial imaging tool for evaluation of patients with an adnexal mass or abnormal uterine bleeding. Ultimately, a number of these are diagnosed with gynecological cancer. Thus, the efficiency of this initial assessment is critical. Although the role of 2D USG in gynecological oncology is well established, it is still far from perfect. Suspicious cases are further investigated with MRI and CT. Surgical examination of the removed specimen is often required for a definitive diagnosis.

Women with ovarian cancer often present with nonspecific symptoms such as dull pelvic/abdominal pain, urinary symptoms, abdominal distention, or bloating. The diagnosis is frequently delayed, increasing the mortality of the disease. Considerable effort has been put into increasing the sensitivity, specificity, and accuracy of 2D USG in differentiating malignant adnexal masses from those that are benign. Several findings such as papillary projections, heterogeneous solid components, thick septae, and low resistance blood flow have been proposed as signs of malignancy. Scoring systems have been developed to increase the sensitivity and specificity of these markers. However, in a meta-analysis, the overall sensitivity and specificity of 2D gray-scale ultrasound was found to be 85.3 and $87.4 \%$, respectively [26]. Development of 3D USG has raised hopes about improving these figures.

Once data about a certain volume are obtained, 3D USG can construct images at any plane. It has the ability to analyze the obtained data using surface rendering, multi-slice imaging, or multi-planar display [27]. Therefore, the examiner can study the mass thoroughly and map it accurately. Volumes of irregular structures are measured precisely using 3D USG. Moreover, it is better at demonstrating septae, cyst wall irregularities, and papillary projections [4]. These qualities help to understand the exact morphology of the adnexal mass. Although several studies did not find 3D grayscale USG to be superior than 2D gray-scale USG [27, 28], the previously referred meta-analysis by Dodge et al. reported an overall sensitivity of $93.5 \%$ and specificity of $91.5 \%$ for 3D gray-scale USG for identifying suspicious adnexal masses [26].

3D USG power Doppler (3D PD) has also been a subject of interest in the evaluation of adnexal masses. Various vascular indices have been proposed. It is believed that vascularization index (VI) represents the amount of vessel in the examined volume, flow index (FI) shows the flow intensity, and vascularization-flow index (VFI) reflects the combination of both [27]. A number of studies examined the suspicious areas in adnexal masses and found that 3D PD indices were significantly higher in those that are malignant [29-32]. It is worth mentioning that there is no agreement among experts on which specific index is better at diagnosing malignancies, or which cut-off value provides the best sensitivity and specificity. Furthermore, the sonographer should be careful about the sampling area, as sampling large areas may cause artifacts and miscalculations. 
Fig. 6 SonoAVC image showing multiple follicles. Each follicle is measured and assigned a different color automatically for easier interpretation. Images in 3 orthogonal planes are combined to form a $3 \mathrm{D}$ image

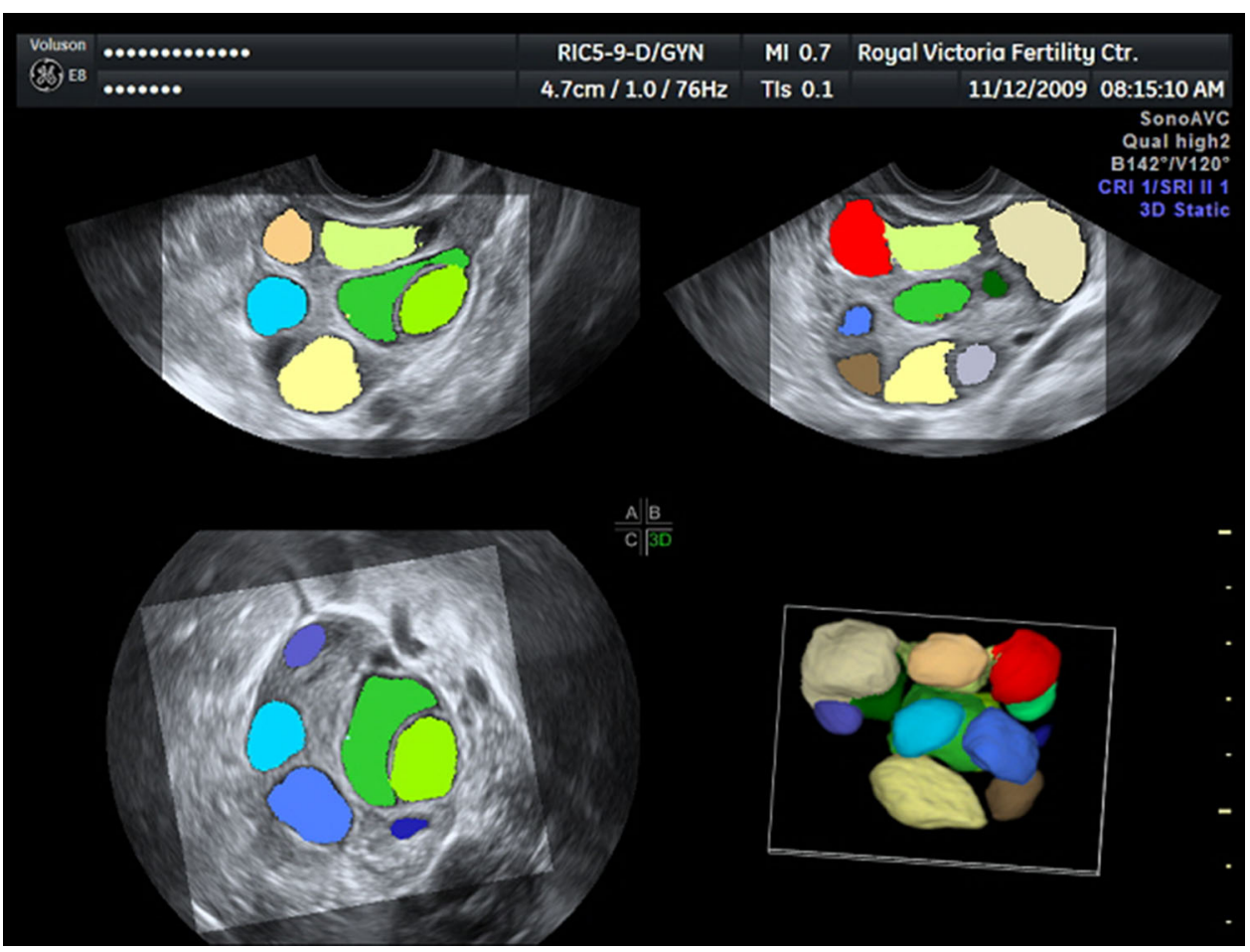

Besides vascular indices, the vascular architecture (or vascular tree) has also been studied as a sign of malignancy. 3D PD can produce a precise model of the vascular architecture and detect these signs. Central vascular flow, septal flow, irregular, and chaotic vascular architecture are associated with malignancy [33]. Kalamantis et al. evaluated 318 women with unilateral adnexal mass using 3D PD. They reported a sensitivity, specificity, positive predictive value, and negative predictive value of $93.5,92.9,84.5$, and $97.2 \%$, respectively [34]. Several other studies provided similar results and concluded that 3D PD is a highly sensitive and specific tool for diagnosing ovarian malignancies [33, 35-37]. 3D USG and 3D PD technique were found to be highly reproducible $[28,38]$.

It can be concluded that 3D USG and 3D PD may provide valuable information about adnexal masses; however, extensive research is required to truly understand their limits and capabilities. If these methods are found to be valuable, this will not only help diagnosing ovarian malignancies and planning accordingly, but also will reduce false positive rates, sparing patients from surgical morbidity, and the psychological burden.

Abnormal uterine bleeding is the most common symptom of endometrial cancer. Measuring the endometrial thickness (ET) with 2D USG is the traditional initial approach on examination. Given that this approach may be inaccurate since it actually misses a dimension, endometrial volume (EV) has been investigated as a criterion. Endometrial volume is measured using virtual organ computer-aided analysis (VOCAL) or a similar software. Studies have shown that EV is a better predictor of endometrial cancer than ET [39-41]. However, each study has defined a different cut-off value for EV, ranging between 1.35 and $3.56 \mathrm{ml}$. Yaman et al. performed 3D endometrial volume measurements on 213 patients with postmenopausal bleeding. After histopathological examination, 42 patients were diagnosed to have endometrial carcinoma. They reported that sensitivity was $100 \%$, specificity $69 \%$, PPV $44 \%$, and NPV $100 \%$ using $2.7 \mathrm{ml}$ as a cut-off value [41]. To improve on these results, researchers added 3D PD to their studies. Vascular indices, namely VI, FI, and VFI, were significantly higher in patients with endometrial carcinoma compared to endometrial hyperplasia [40, 42, 43]. The difference was even more prominent when myometrial invasion was more than $50 \%$ [43]. Although these results are interesting, they lack the power to change the current practice.

The role of 3D USG in gynecologic oncology is not well established. Studies so far are promising yet weak and inconsistent. Their capabilities and weaknesses should be examined further. Application of 3D USG techniques and interpretation of the results should be standardized. Unlike its definitive diagnostic role in benign gynecology, 3D USG is mainly used for the initial evaluation of patients with potential malignancy. 


\section{Urogynecology}

Of all the subspecialties in obstetrics and gynecology, urogynecology has been the last one to implement ultrasound in its routine practice. The primary methods in diagnosing pelvic floor disorders have been physical examination and urodynamic tests. 2D translabial ultrasound (2D TL), MRI, and dynamic MRI have been used as imaging tools for the evaluation of urogynecology patients. However, each has their limitations. 2D TL does not have access to the axial plane, which reduces its capacity to show the pelvic floor structures. MRI can show the axial plane, but it is expensive, not readily available and time consuming. Moreover, due to the physical properties of the device, required maneuvers cannot always be performed by the patient during dynamic MRI [44].

The introduction of translabial 3D USG (3D TL) has changed the approach to imaging technologies in urogynecology. It has become an invaluable tool for clinicians for a number of reasons. First of all, it provides instant access to the axial plane. This plane clearly depicts the relationship of the vagina, urethra, rectum, and the muscular pelvic floor. Unlike MRI, acquired volumes can be examined in any desired plane, which increases accessibility to some structures in challenging locations [45]. It also provides real-time images with acceptable quality, which can give information about the function of the examined tissues, i.e., prolapse or mobility. Multi-slice imaging can effectively detect and measure the size of pelvic floor defects such as levator ani injuries [46]. Moreover, it is found to be highly reproducible [47]. Several studies reported that there were no statistically significant differences between 3D TL and MRI measurements made for the evaluation of the pelvic floor, and 3D TL may end the need for MRI evaluation [48-50].

Three-D TL can also be used for locating tension-free sub-uretheral slings that are used in the treatment of stress urinary incontinence. These slings are hard to locate using MRI; however, they are easy to spot on ultrasound since they are hyperechogenic [4]. Likewise, synthetic meshes, such as vaginal wall meshes, are easily seen using 3D TL. Determining the location of the sling or the mesh and observing it in real time help the surgeon to assess the efficacy of his treatment [47].

\section{Disadvantages of 3D USG}

3D USG equipment is not as widely available as 2D USG, resulting in limited access to the device. Therefore, clinicians are more experienced in 2D USG. Manipulating a volume can be a cumbersome and time-consuming process for the inexperienced user. This may result in an inclination to stay on safe ground and not making full use of the 3D USG technology.

The volume data obtained using 3D USG are remarkably large. Archiving, sharing, or transferring can be an issue, especially in busy clinics.

Last of all, it should be remembered that although 3D USG produces less artifacts than 2D USG, as all imaging techniques, 3D USG is not artifact-free.

\section{Conclusion}

3D USG is a fast-evolving imaging technique that holds a great potential for use in gynecology. It is not hard to imagine that as more clinicians gain access to 3D USG and improve their skills, 3D USG will prove to be an even more powerful diagnostic device. It provides quick and high-quality access to planes that were previously unavailable. Different branches of gynecology make use of these new opportunities. Evaluation of congenital uterine anomalies and urogynecology are the two areas where 3D USG is successful enough to replace previous imaging modalities. However, more studies are needed to discover its capabilities in oncology.

In conclusion, clinicians can benefit in adding 3D USG to their routine gynecological workup as it provides fast and accurate results in a relatively cost-effective setting.

Conflict of Interest The authors declare no conflict of interest.

\section{References}

1. Chan YY, Jayaprakasan K, Zamora J, et al. The prevalence of congenital uterine anomalies in unselected and high-risk populations: a systematic review. Hum Reprod Update. 2011;17(6): 761-71.

2. Saravelos SH, Cocksedge KA, Li TC. Prevalence and diagnosis of congenital uterine anomalies in women with reproductive failure: a critical appraisal. Hum Reprod Update. 2008;14(5): 415-29.

3. Chan YY, Jayaprakasan K, Tan A, et al. Reproductive outcomes in women with congenital uterine anomalies: a systematic review. Ultrasound Obstet Gynecol. 2011;38(4):371-82.

4. Armstrong L, Fleischer A, Andreotti R. Three-dimensional volumetric sonography in gynecology: an overview of clinical applications. Radiol Clin North Am. 2013;51(6):1035-47.

5. Faivre E, Fernandez H, Deffieux X, et al. Accuracy of threedimensional ultrasonography in differential diagnosis of septate and bicornuate uterus compared with office hysteroscopy and pelvic magnetic resonance imaging. J Minim Invasive Gynecol. 2012;19(1):101-6.

6. Benacerraf BR, Shipp TD, Bromley B. Which patients benefit from a $3 \mathrm{D}$ reconstructed coronal view of the uterus added to standard routine 2D pelvic sonography? AJR Am J Roentgenol. 2008;190(3):626-9.

7. Ghi T, et al. Accuracy of three-dimensional ultrasound in diagnosis and classification of congenital uterine anomalies. Fertil Steril. 2009;92(2):808-13. 
8. Bermejo C, Martinez Ten P, Cantarero R, et al. Three-dimensional ultrasound in the diagnosis of Mullerian duct anomalies and concordance with magnetic resonance imaging. Ultrasound Obstet Gynecol. 2010;35(5):593-601.

9. Ata B, Nayot D, Nedelchev A, et al. Do measurements of uterine septum using three-dimensional ultrasound and magnetic resonance imaging agree? J Obstet Gynaecol Can. 2014;36(4):331-8.

10. Ludwin A, Pityński K, Ludwin I, et al. Two- and three-dimensional ultrasonography and sonohysterography versus hysteroscopy with laparoscopy in the differential diagnosis of septate, bicornuate, and arcuate uteri. J Minim Invasive Gynecol. 2013;20(1):90-9.

11. Ludwin A, Ludwin I, Kudla M, et al. Diagnostic accuracy of three-dimensional sonohysterography compared with office hysteroscopy and its interrater/intrarater agreement in uterine cavity assessment after hysteroscopic metroplasty. Fertil Steril. 2014;101(5):1392-9.

12. Ludwin A, Ludwin A, Pitynski $\mathrm{K}$ et al. Role of morphologic characteristics of the uterine septum in the prediction and prevention of abnormal healing outcomes after hysteroscopic metroplasty. Hum Reprod. 2014.

13. Bragg AC, Angtuaco TL. Three-dimensional gynecologic ultrasound. Ultrasound Clinics. 2010;5(2):299-311.

14. Abou-Salem N, Elmazny A, El-Sherbiny W. Value of 3-dimensional sonohysterography for detection of intrauterine lesions in women with abnormal uterine bleeding. J Minim Invasive Gynecol. 2010;17(2):200-4.

15. Knopman J, Copperman AB. Value of 3D ultrasound in the management of suspected Asherman's syndrome. J Reprod Med. 2007;52(11):1016-22.

16. Ahmadi F, Javam M. Role of 3D sonohysterography in the investigation of uterine synechiae/asherman's syndrome: pictorial assay. J Med Imaging Radiat Oncol. 2014;58(2):199-202.

17. Exacoustos C, Brienza L, Di Giovanni A, et al. Adenomyosis: three-dimensional sonographic findings of the junctional zone and correlation with histology. Ultrasound Obstet Gynecol. 2011;37(4):471-9.

18. Luciano DE, Exacoustos C, Albrecht L, et al. Three-dimensional ultrasound in diagnosis of adenomyosis: histologic correlation with ultrasound targeted biopsies of the uterus. J Minim Invasive Gynecol. 2013;20(6):803-10.

19. Benacerraf BR, Shipp TD, Bromley B. Three-dimensional ultrasound detection of abnormally located intrauterine contraceptive devices which are a source of pelvic pain and abnormal bleeding. Ultrasound Obstet Gynecol. 2009;34(1):110-5.

20. Bazot M, Lafont C, Rouzier R, et al. Accuracy of transvaginal sonography and rectal endoscopic sonography in the diagnosis of deep infiltrating endometriosis. Ultrasound Obstet Gynecol. 2007;30(7):994-1001.

21. Guerriero S, Ajossa S, Gerada M, et al. "Tenderness-guided" transvaginal ultrasonography: a new method for the detection of deep endometriosis in patients with chronic pelvic pain. Fertil Steril. 2007;88(5):1293-7.

22. Guerriero S, Saba L, Ajossa S, et al. Three-dimensional ultrasonography in the diagnosis of deep endometriosis. Hum Reprod. 2014;29(6):1189-98.

23. Raine-Fenning N, Jayaprakasan K, Deb S, et al. Automated follicle tracking improves measurement reliability in patients undergoing ovarian stimulation. Reprod Biomed Online. 2009; 18(5):658-63.

24. Ata B, Tulandi T. Ultrasound automated volume calculation in reproduction and in pregnancy. Fertil Steril. 2011;95(7):2163-70.

25. Ata B, Seyhan A, Reinblatt SL, et al. Comparison of automated and manual follicle monitoring in an unrestricted population of 100 women undergoing controlled ovarian stimulation for IVF. Hum Reprod. 2011;26(1):127-33.
26. Dodge JE, Covens AL, Lacchetti C, et al. Preoperative identification of a suspicious adnexal mass: a systematic review and meta-analysis. Gynecol Oncol. 2012;126(1):157-66.

27. Alcazar JL, Jurado M. Three-dimensional ultrasound for assessing women with gynecological cancer: a systematic review. Gynecol Oncol. 2011;120(3):340-6.

28. Alcazar JL, Garcia-Manero M, Galvan R. Three-dimensional sonographic morphologic assessment of adnexal masses: a reproducibility study. J Ultrasound Med. 2007;26(8):1007-11.

29. Alcazar JL, Rodriguez D. Three-dimensional power Doppler vascular sonographic sampling for predicting ovarian cancer in cystic-solid and solid vascularized masses. J Ultrasound Med. 2009;28(3):275-81.

30. Geomini PM, Coppus SF, Kluivers KB, et al. Is three-dimensional ultrasonography of additional value in the assessment of adnexal masses? Gynecol Oncol. 2007;106(1):153-9.

31. Kudla MJ, Alcazar JL. Does sphere volume affect the performance of three-dimensional power Doppler virtual vascular sampling for predicting malignancy in vascularized solid or cystic-solid adnexal masses? Ultrasound Obstet Gynecol. 2010;35(5):602-8.

32. Kudla MJ, Timor-Tritsch IE, Hope JM, et al. Spherical tissue sampling in 3-dimensional power Doppler angiography: a new approach for evaluation of ovarian tumors. J Ultrasound Med. 2008;27(3):425-33.

33. Chase DM, Crade M, Basu T, et al. Preoperative diagnosis of ovarian malignancy: preliminary results of the use of 3-dimensional vascular ultrasound. Int J Gynecol Cancer. 2009;19(3):354-60.

34. Kalmantis K, Rodolakis A, Daskalakis G, et al. Characterization of ovarian tumors and staging ovarian cancer with 3-dimensional power Doppler angiography: correlation with pathologic findings. Int J Gynecol Cancer. 2013;23(3):469-74.

35. Alcazar JL, Iturra A, Sedda F, et al. Three-dimensional volume off-line analysis as compared to real-time ultrasound for assessing adnexal masses. Eur $\mathrm{J}$ Obstet Gynecol Reprod Biol. 2012;161(1):92-5.

36. Kalmantis K, Papageorgiou T, Rodolakis A, et al. The role of three-dimensional (3D) sonography and 3D power Doppler in the preoperative assessment of borderline ovarian tumors. Eur $\mathrm{J}$ Gynaecol Oncol. 2007;28(5):381-5.

37. Laban M, Metawee H, Elyan A, et al. Three-dimensional ultrasound and three-dimensional power Doppler in the assessment of ovarian tumors. Int J Gynaecol Obstet. 2007;99(3):201-5.

38. Guerriero S, Saba L, Ajossa S, et al. Assessing the reproducibility of the IOTA simple ultrasound rules for classifying adnexal masses as benign or malignant using stored 3D volumes. Eur J Obstet Gynecol Reprod Biol. 2013;171(1):157-60.

39. Mansour GM, El-Lamie IKI, El-Kady MA, et al. Endometrial volume as predictor of malignancy in women with postmenopausal bleeding. Int J Gynaecol Obstet. 2007;99(3):206-10.

40. Odeh M, Vainerovsky I, Grinin V, et al. Three-dimensional endometrial volume and 3-dimensional power Doppler analysis in predicting endometrial carcinoma and hyperplasia. Gynecol Oncol. 2007;106(2):348-53.

41. Yaman C, Habelsberger A, Tews G, et al. The role of threedimensional volume measurement in diagnosing endometrial cancer in patients with postmenopausal bleeding. Gynecol Oncol. 2008;110(3):390-5.

42. Alcazar JL and Galvan R. Three-dimensional power Doppler ultrasound scanning for the prediction of endometrial cancer in women with postmenopausal bleeding and thickened endometrium. Am J Obstet Gynecol. 2009. 200(1): p. 44 e1-6.

43. Merce LT, Alcazar JL, Lopez C, et al. Clinical usefulness of 3-dimensional sonography and power Doppler angiography for diagnosis of endometrial carcinoma. J Ultrasound Med. 2007;26(10):1279-87. 
44. Dietz HP, Shek C. Levator avulsion and grading of pelvic floor muscle strength. Int Urogynecol J Pelvic Floor Dysfunct. 2008;19(5):633-6.

45. Dietz HP. The role of two- and three-dimensional dynamic ultrasonography in pelvic organ prolapse. J Minim Invasive Gynecol. 2010;17(3):282-94.

46. Schwertner-Tiepelmann N, Thakar R, Sultan AH, et al. Obstetric levator ani muscle injuries: current status. Ultrasound Obstet Gynecol. 2012;39(4):372-83.

47. Dietz HP. Pelvic floor ultrasound: a review. Am J Obstet Gynecol. 2010;202(4):321-34.

48. Kruger JA, Heap SW, Murphy BA, et al. Pelvic floor function in nulliparous women using three-dimensional ultrasound and magnetic resonance imaging. Obstet Gynecol. 2008;111(3): 631-8.

49. Majida M, Braekken IH, Bo K, et al. Validation of threedimensional perineal ultrasound and magnetic resonance imaging measurements of the pubovisceral muscle at rest. Ultrasound Obstet Gynecol. 2010;35(6):715-22.

50. Zhuang RR, Song YF, Chen ZQ et al. Levator avulsion using a tomographic ultrasound and magnetic resonance-based model. Am J Obstet Gynecol. 2011. 205(3): p. 232 e1-8. 